\title{
Velocity of head growth during the perinatal period
}

\author{
MASANORI FUJIMURA AND JUN-ICHI SERYU \\ From the Departments of Paediatrics and Obstetrics and Gynaecology, Osaka University Medical School, \\ and Department of Paediatrics, Palmore Hospital, Kobe, Japan
}

SUMMARY Growth velocity of head circumference was studied longitudinally in different gestational age groups of 222 appropriate-weight-for-dates (AFD) and 94 small-for-dates (SFD) healthy infants during the first 5 months of life. Term AFD and SFD infants showed a steady slowing of growth rate of head circumference from birth. In contrast, preterm AFD infants of less than 36 weeks' gestation showed an increasing velocity of growth followed by slowing, with maximum velocity occurring between 30 and 40 days after birth. The shorter the postconceptional age at birth the later maximum velocity occurred. However, those preterm AFD infants of between 30 and 33 weeks' gestation who were given a high caloric feed showed a similar velocity curve to that of infants of 34-37 weeks of gestation.

Cross-sectional data were used to estimate growth velocity of head circumference in the fetus. Two conclusions emerged. First, there is a slowing of head growth velocity from 31 weeks' gestation in utero, and second, term infants show a marked increase in velocity after birth. Though the occurrence of maximum velocity of head growth is delayed in the preterm infant, the net effect is such that at a given postconceptional age his head circumference is greater than that of the term infant, at least within the first 5 postnatal months.

The intellectual and neurological development of low birthweight infants is a cause for great concern. Although intensive care of these neonates has reduced the incidence of severe neurological deficits (Hagberg et al., 1973; Davies and Stewart, 1975; Davies and Tizard, 1975), evidence of minor cerebral dysfunction occurs in the form of learning difficulties and poor school performance (Davies and Stewart, 1975).

The human brain grows most rapidly during the perinatal period (Dobbing and Sands, 1973) and therefore pathological conditions occurring at this time would be more likely to damage development and function of the brain than at any other time. The concept of 'vulnerability' or 'critical period' is well established (Muralt, 1972).

There are good reasons to believe that during infancy and childhood the increase in occipitofrontal head circumference (OFC) correlates well with the growth of the intracranial volume and the brain (Bray et al., 1969; Winick and Rosso, 1969; Buda et al., 1975). Small head size has been associated with poor intellectual performance by some authors (O'Connell et al., 1965; Pryor and Thelander, 1968; Babson and Henderson, 1974; Francis-Williams and Davies, 1974).

Received 15 May 1976
Few studies have been done on the growth velocity of head circumference during the specific period of neonatal and infant life. O'Neill (1961) used a growth curve of head circumference of preterm infants during the neonatal period for the early detection of hydrocephalus. This curve showed an increased rate of head growth compared with the curve of the term infant. Davies and Davis (1970) showed a mean daily head growth rate of 0.08 $0.10 \mathrm{~cm}$ for preterm infants during the neonatal period. Stunted growth of the head was associated with a relatively low food intake and low body temperature during the first week of life.

Cruise (1973) studied weight, length, and head circumference during the first 8 years of life in 202 low birthweight and 113 term appropriate-weightfor-dates (AFD) infants. The velocity of growth of head circumference from birth to 13 weeks of life was found to be larger $(0.10 \mathrm{~cm} / \mathrm{d})$ in the preterm infants compared to those of more mature weight or those small-for-dates (SFD) $(0.09 \mathrm{~cm} / \mathrm{d}$ and $0.08 \mathrm{~cm} / \mathrm{d}$, respectively). Glass et al. (1975) studied the effect of thermal environment and caloric intake on head growth of low birthweight infants during the neonatal period. The rates of increase in head circumference were from 0.13 to $0.15 \mathrm{~cm} /$ day over the 2-week period. Exposure to a sub- 
thermoneutral temperature together with a relatively low calorie intake was associated with significant retardation of head growth. Recently, Sher and Brown $(1975 \mathrm{a}, \mathrm{b})$ reported a longitudinal study of head growth in preterm infants. Normal rates of head growth of healthy preterm infants of 30-33 weeks, 34-37 weeks' gestation, and sick preterm infants of various gestational ages were shown for each week after birth during the first 4 weeks, with longer intervals thereafter until the age of 16 weeks. The most rapid rates of growth occurred in the smallest infants. Neonates with significant medical problems conform to or fall below the expected patterns of growth.

From those studies the preterm infants were shown to have a higher rate of head growth compared with the more mature infants during the neonatal period. It is still not clear how the rate of growth of head circumference changes as age increases in relation to gestational age at birth. This study examines the relationship of gestational age at birth and the patterns of growth velocity of the head circumference in normal term and preterm infants.

\section{Patients and methods}

Infants in the preterm and newborn unit of Palmore Hospital, Kobe, who were followed up longitudinally for at least 6 months at the hospital from 1971 to 1973 were studied. Excluded from the study were those of uncertain gestational age, those with a history of serious illness including the respiratory distress syndrome and cranial pathology, those with OFC measurements recorded less than four times in the first 6 months of life, and those with evidence of gross neurological defects or retardation. All infants were alive at the time of analysis.

Those with birthweights between the 10th and 90th centiles of the intrauterine growth chart (Lubchenco et al., 1966) were classified as AFD and those less than the 10th centile as SFD. Gestational age was counted in completed weeks after the first day of the last menstrual period. Randomly selected AFD infants of 36-41 weeks' gestation with birthweights between the 25th and 75th centiles and all AFD infants of 30-35 weeks' gestation were divided into groups $\mathrm{A}, \mathrm{C}, \mathrm{D}$, and $\mathrm{E}$ according to gestational age (Table 1). All SFD infants born between 38 and 41 weeks' gestation were studied (group B).

All measurements of OFC and body weight were done by nurses using routine standardized methods on the first day of life, and then once a week in the nursery and once in every 2 or 3 months up to 12 months of age after discharge at the hospital clinic. Tape measures used were made of cloth covered with plastic. Each infant was weighed on a balance accurate to the nearest $10 \mathrm{~g}$. 6 nurses made the measurements, but more than $75 \%$ were done by 2 nurses only.

The growth curves of OFC and body weight as functions of age in days were estimated by a curve fitting procedure from the longitudinal data; for each group of infants the polynomial regression was estimated by the method of least squares by the computer. The rate of daily increase (growth velocity) of OFC and body weight were estimated by differentiation of the growth function. For the estimation of intrauterine head growth, a regression curve was obtained by the same method from the cross-sectional data of OFC at birth and gestational age.

Postnatal change of the shape of skull in low birthweight infants (Baum and Searls, 1971; Lengerke, 1974) was considered as a possible factor of error for the velocity analysis. The difference between real and corrected OFC during the first year was reported to be approximately $10 \mathrm{~mm}$ at the maximum, which is of the order of $10^{-2} \mathrm{~mm}$ for the daily velocity value and can be neglected for purposes of this study.

\section{Results*}

Intrauterine growth and velocity of OFC. Estimated growth of head circumference during intrauterine

*The mathematical formulae for constructing the curves of head growth with postnatal age in each of the 8 groups may be obtained from the author.

Table 1 Details of babies studied

\begin{tabular}{lllll}
\hline Group & $\begin{array}{l}\text { Gestational age } \\
\text { in completed weeks }\end{array}$ & $\begin{array}{l}\text { Range of birthweight } \\
\text { on intrauterine growth } \\
\text { chart* (centile) }\end{array}$ & $\begin{array}{l}\text { No. of } \\
\text { cases }\end{array}$ & $\begin{array}{l}\text { Birthweight }(\mathrm{g}) \\
(\text { mean } \pm S D)\end{array}$ \\
\hline A & $38-41$ & $25-75$ & 93 & $3159 \pm 321$ \\
B & $38-41$ & $<10$ & 94 & $2352 \pm 164$ \\
C & $36 / 37$ & $25-75$ & 49 & $2852 \pm 244$ \\
D & $34 / 35$ & $10-90$ & 39 & $2337 \pm 267$ \\
E & $30-33$ & $10-90$ & 41 & $1845 \pm 253$ \\
\hline
\end{tabular}

* Based on the intrauterine growth chart, Lubchenco et al. (1966).

OFC $=$ occipitofrontal head circumference. 
life is shown in Fig. 1a, which is based on head circumference measurements made at birth on AFD term and preterm babies of known gestational ages (groups A, C, D, E, and 6 additional cases of 27-29 weeks' gestation). Daily velocity of OFC growth decreases after 31 weeks of gestation (Fig. 2a).

OFC growth of term AFD and SFD infants. Fig. 1b gives the postnatal OFC growth of term AFD and term SFD infants. Though the absolute value of head growth was different in the two groups, the pattern of growth velocity which showed rapidly decreasing speed of head growth after birth was similar (Fig. 2b). Figs. 3 and 4 give the sex difference in the absolute growth and velocity growth of OFC. A single intrauterine growth chart was used for the selection of these cases; thus the number of SFD females was twice as large as that of SFD males, but this did not bias the combined velocity curve of male and female of Fig. $2 b$.

OFC growth of preterm infants. In Figs. 5 and 6, the postnatal head growth in terms of absolute values and of the velocity of growth are shown for infants in groups A, C, D, and E. In Fig. 6, infants in groups $D$ and $E$ showed characteristic peak curves of velocity of growth after birth with the maximum velocity between 4 and 6 weeks of age, decreasing gradually after that though maintaining relatively high growth velocity up to 20 weeks after birth. Infants of 36 and 37 weeks' gestation (group C) showed an intermediate pattern between term and preterm infants.

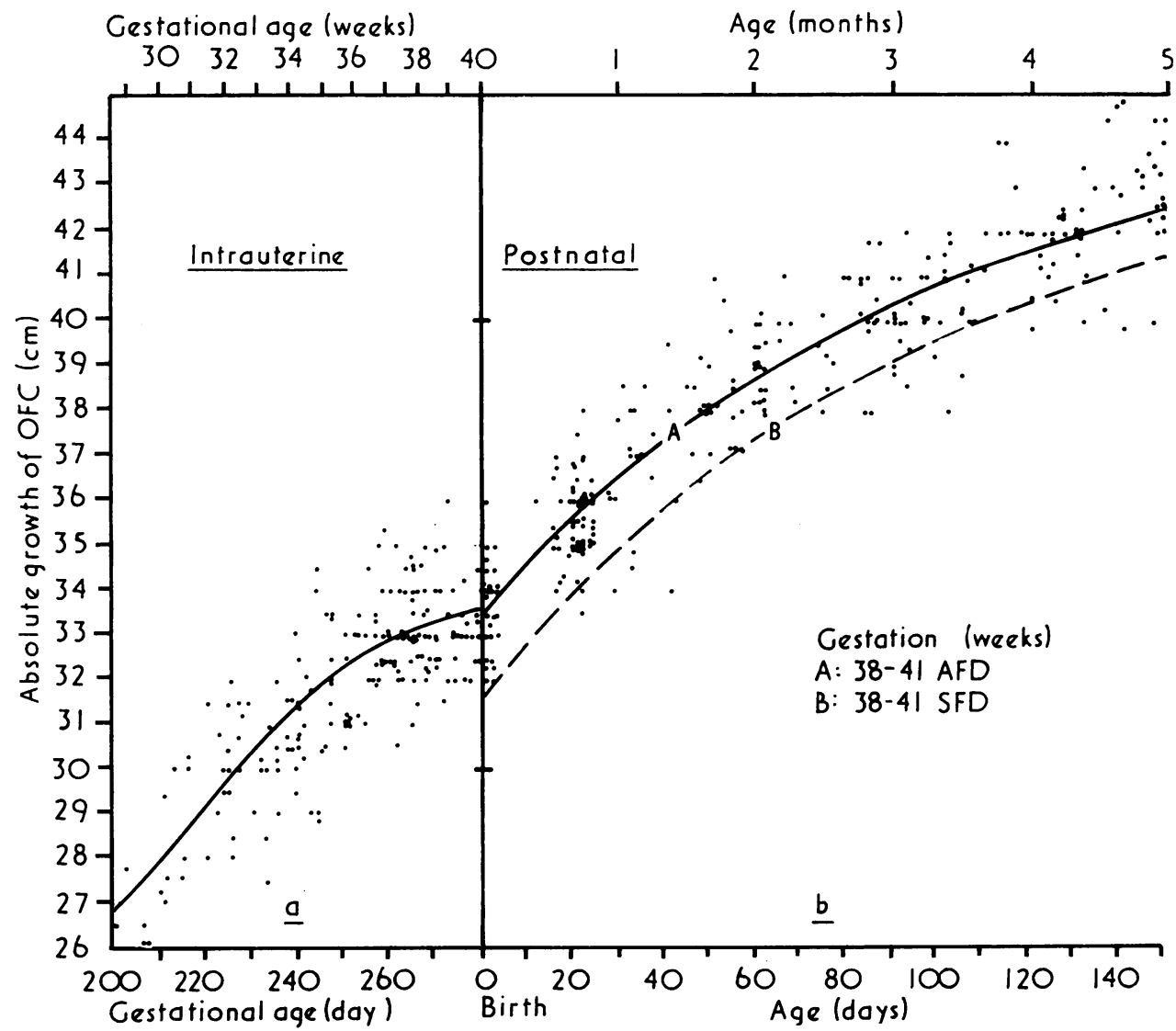

Fig. 1 Absolute growth of OFC. (a) Cross-sectional intrauterine growth estimated from the OFC of AFD babies at birth (groups $A, C, D, E$, and 6 additional cases of 27-29 weeks' gestation). Individual cases are plotted. $n=231$; $1 S D=1.46 \mathrm{~cm} ; r=0 \cdot 57$. (b) Postnatal growth of term babies (groups $A$ and $B$ ). Individual cases are plotted for group $A$. $A: n=515 ; 1 S D=1 \cdot 13 \mathrm{~cm} ; r=0.93$. $B: n=681,1 S D=1 \cdot 05 \mathrm{~cm} ; r=0 \cdot 92 . A F D=$ appropriate for dates; $S F D=$ small for dates. 


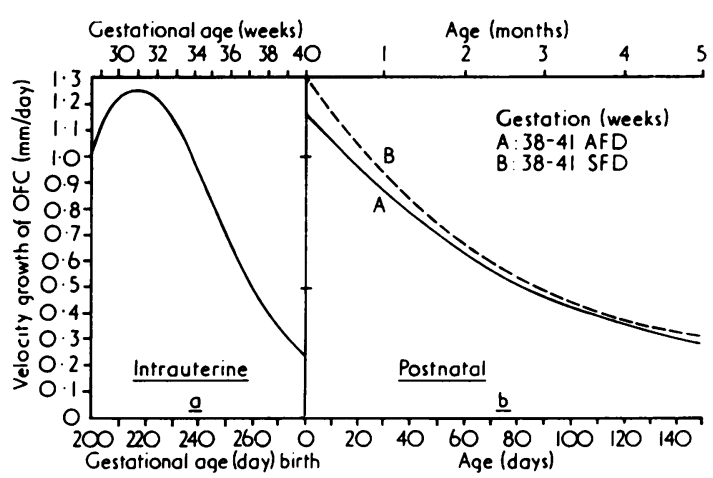

Fig. 2 Daily velocity growth of OFC. (a) Intrauterine velocity growth estimated from the absolute growth curve of OFC in Fig. 1a. (b) Postnatal velocity growth of term babies (groups $A$ and $B$ ) estimated from the absolute growth curve of OFC in Fig. $1 b$.

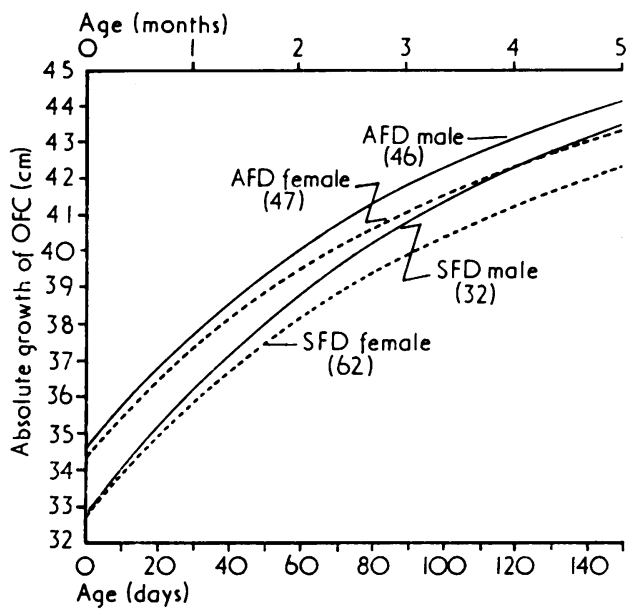

Fig. 3 Comparison of absolute growth of OFC in both sexes of term babies (groups $A$ and B). Both males and females were selected based on the single intrauterine growth chart so that the similar values of the size at birth in both sexes are observed. Number of cases in parentheses.

OFC growth of babies in two subgroups of group $E$. From the above observations, the velocity of postnatal OFC growth seems to be affected by the gestational age at birth. Considering the fact that body temperature and nutrition may influence postnatal head growth (Davies and Davis, 1970; Glass et al., 1975), the possibility exists that some of the small preterm infants studied might show differences in the velocity of OFC growth from infants of the same gestational age. We examined the possible effect of feeding in the following way. The caloric

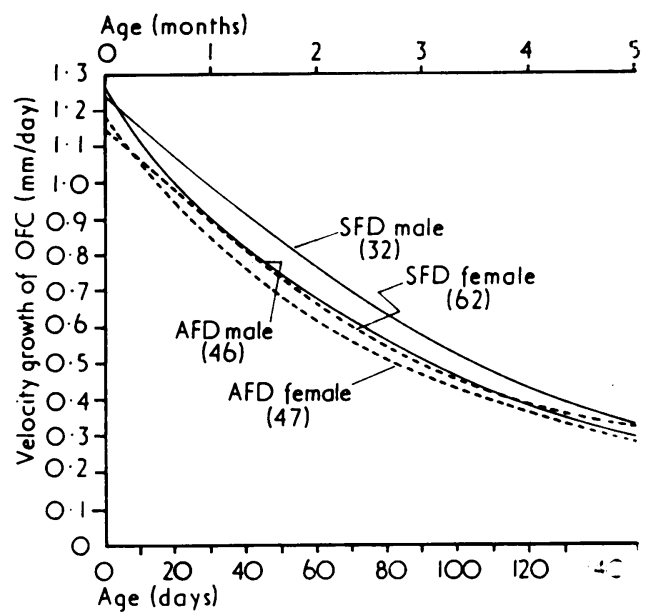

Fig. 4 Comparison of daily velocity growth of OFC in both sexes of term babies (groups $A$ and B). Number of cases in parentheses.

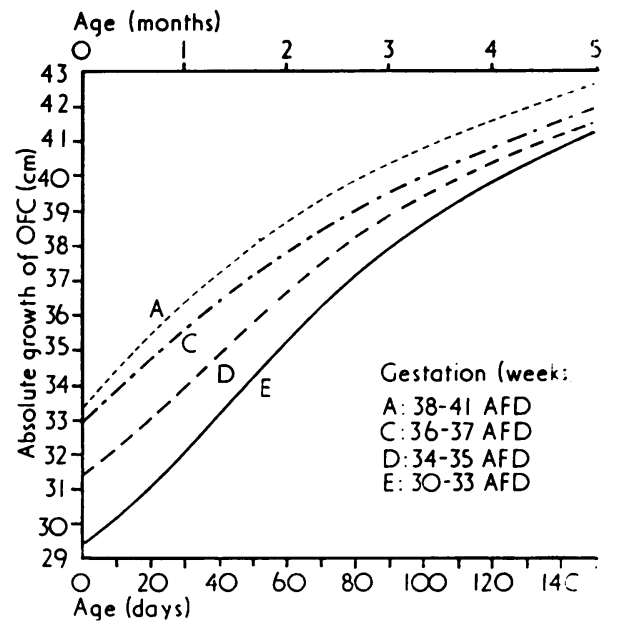

Fig. 5 Absolute growth of OFC of different gestational age groups (groups $A, C, D$, and $E$ ). $C: n=316$; I $S D=0.89 \mathrm{~cm} ; r=0.96 . D: n=281 ; 1 S D=1 \cdot 21$ $\mathrm{cm} ; r=0.94$. $E: n=282 ; 1 S D=1.44 \mathrm{~cm} ; r=0.94$.

intake per $\mathrm{kg}$ body weight over a 24-hour period on the 30th postnatal day was recorded in 41 infants in group E. Half had an intake of more than $130 \mathrm{kcal} /$ $\mathrm{kg}(543 \mathrm{~kJ})$ (group H) and this group was compared with the remainder (group L). Mean birthweight and head circumference at birth were not different in both groups (Table 2 ).

The absolute growth of OFC in groups $\mathrm{H}$ and $\mathrm{L}$ is shown in Fig. 7. The growth velocity showed a difference in that the pattern of infants in group $\mathrm{H}$ 
Table 2 Physical measurements of babies in group $E$ at birth (mean $\pm 1 S D)$

\begin{tabular}{llll}
\hline Group & $\begin{array}{l}\text { No. of } \\
\text { cases }\end{array}$ & Birthweight (g) & OFC at birth $(\mathrm{cm})$ \\
\hline H & 21 & $1870 \pm 216^{*}$ & $\begin{array}{l}29 \cdot 5 \pm 1 \cdot 5^{*} \\
\text { L }\end{array}$ \\
\hline
\end{tabular}

*Not significantly different.

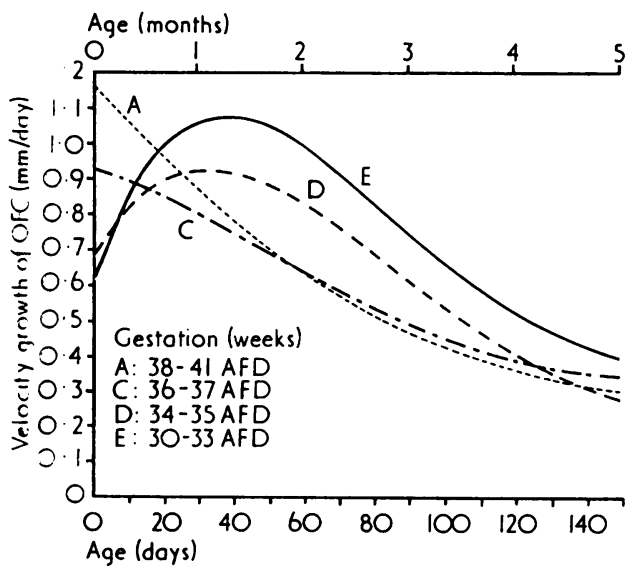

Fig. 6 Daily velocity growth of OFC of different gestational age groups (groups $A, C, D$, and $E$ ).

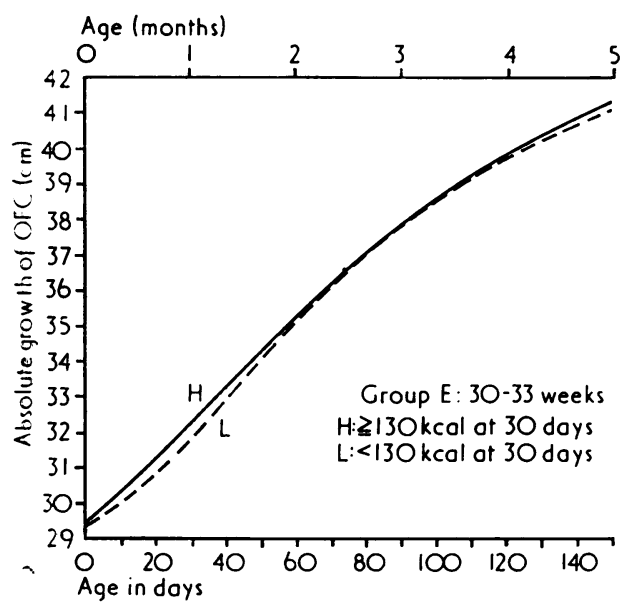

Fig. 7 Absolute growth of OFC of babies between 30 and 33 weeks' gestation (group E). At the age of 30 days babies in group $H$ were receiving $\geqslant 130 \mathrm{kcal} / \mathrm{kg}$ per day $(543 \mathrm{~kJ})$, and in group $L$ the caloric intake was less. $H: n=148,1 S D=1 \cdot 23 \mathrm{~cm} ; r=0 \cdot 95 . L$ : $n=134 ; 1 S D=1.59 \mathrm{~cm} ; r=0.93$. (higher calorie intake) was similar to that of infants in group $\mathrm{D}$ who were more mature in gestation (Fig. 8).

Growth of OFC on the postconceptional scale. In Figs. 9 and 10, the curves of head growth of the infants were summarized on the postconceptional scale. Curves of preterm infants, either absolute or velocity of growth, differed from what they would have been if infants had been born at term. On the absolute growth of OFC, they showed a short period of suppression of growth soon after birth, then an overshoot, followed by an approximation to the

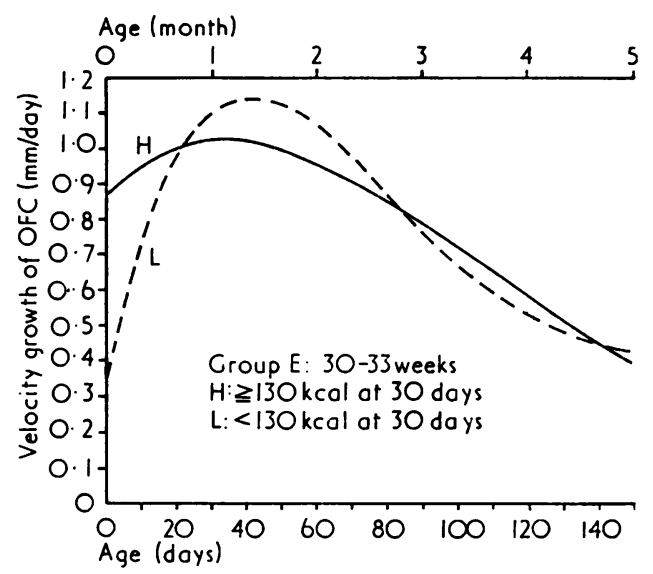

Fig. 8 Daily velocity grcwth of OFC of babies between 30 and 33 weeks' gestation (group E). At the age of 30 days babies in group $H$ were receiving $\geqslant 130 \mathrm{kcal} / \mathrm{kg}$ per day $(543 \mathrm{~kJ})$, and in group $L$ the caloric intake was less.

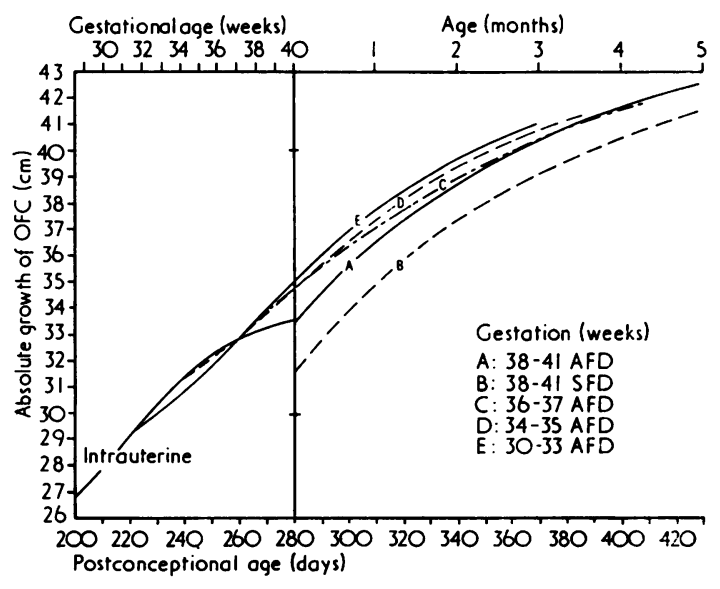

Fig. 9 Absolute growth of OFC of five groups against postconceptional age. 


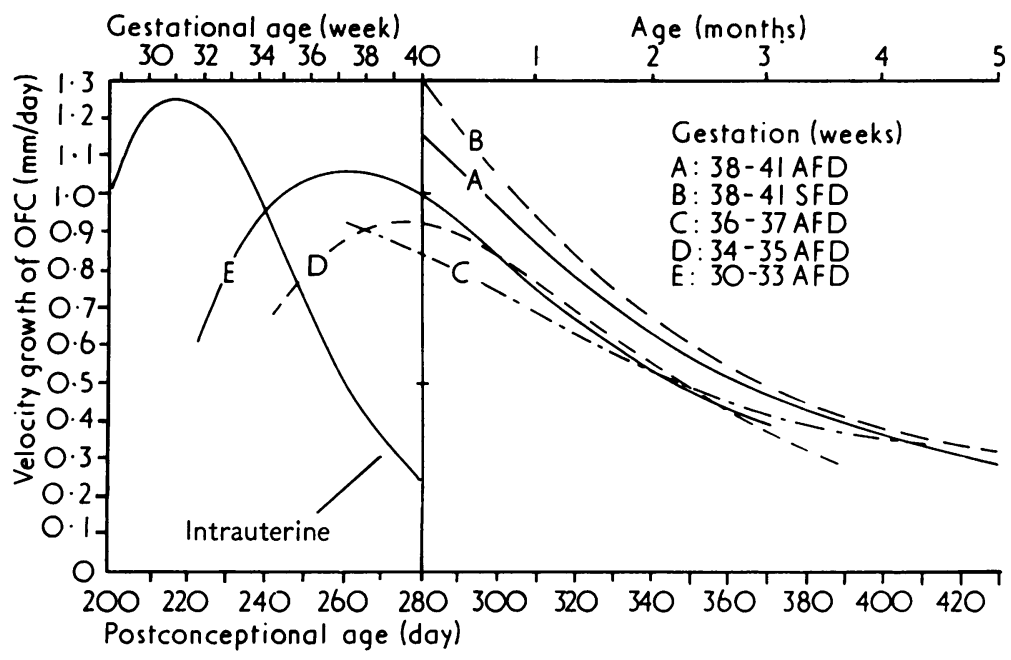

Fig. 10 Daily velocity growth of OFC of five groups against postconceptional age.

growth curve of term infants. The difference in OFC at 40 weeks conceptional age between groups $C$, $\mathrm{D}, \mathrm{E}$ and group $\mathrm{A}$ were significant $(\mathrm{A}-\mathrm{C}, \mathrm{P}<0.001$; A-D, P $<0.05 ;$ A-E, P $<0.01)$.

Growth of body weight. Figs. 11 and 12 show the postnatal growth in weight in groups A, B, C, D, and $\mathrm{E}$, respectively. In comparison with the OFC growth as already shown, several points can be seen. First, in AFD infants, as seen on the velocity

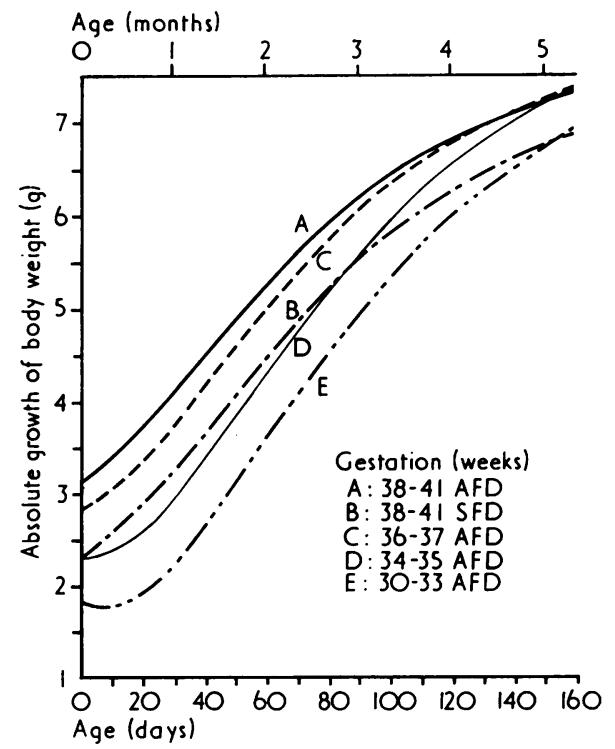

Fig. 11 Absolute growth of body weight of different gestational age groups.

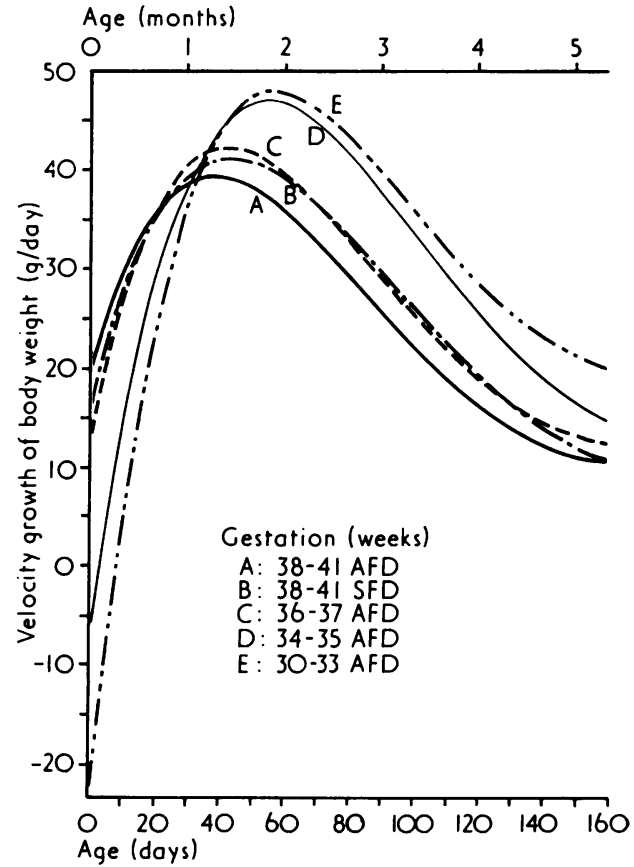

Fig. 12 Daily velocity growth of body weight of different gestational age groups.

curves of OFC (Fig. 6), the younger the postconceptional age at birth, the lower the initial velocity of weight growth. Second, the timing of maximum velocity is different in the two measures of physical growth. The timing comes earlier for OFC than body weight in each group. Finally, the smaller the 
infants at birth the higher the velocity of growth after they reached the peak, and this continues at least until 5 months of age.

\section{Discussion}

In this paper graphs of growth velocity of OFC are shown as functions of postnatal age in days in groups of different gestational ages. Although very few studies have been published on the velocity growth of OFC during the perinatal period, comparison of intrauterine velocity growth of OFC in one study (P. Davies, personal communication, 1974) showed identical curves for absolute value and shape, even though the method of estimation of the curves was quite different. Weekly increment of OFC and brain weight during the last trimester, calculated by the authors from other sources (Lubchenco et al., 1966; Gruenwald, 1967), also showed similar results. Our observation that the peak velocity of OFC occurs at 31 weeks' gestation agrees with an analysis by Cheek (1975) using cross-sectional data for brain weight. Interestingly, this is the time when the percentage of brain weight in relation to body weight starts to decrease and when certain biochemical changes-probably connected with myelinationappear abruptly (Conde et al., 1974).

Term AFD infants show a steady deceleration of head growth during the last 9 weeks of gestation (Fig. 2a). In contrast, the same group achieve maximum head growth velocity shortly after birth, thereafter decreasing with age (Fig. 2b). The discrepancy between growth rate at term and that just after birth is striking. Obviously, the decelerating trend of the velocity curve could not be extended straight forward in the term infants, as the velocity is so low at that time that no growth could be expected soon after birth. It has been a well known observation in preterm infants followed longitudinally that OFC, if plotted on the intrauterine growth chart, grows crossing over the upper centile lines. This fact combined with the above observations on term infants indicates suppression of head growth during late gestation in utero, with release at birth. It has been claimed that birth is only of passing importance to brain development (Dobbing, 1974). Our findings seem to contradict this.

In preterm infants patterns of head growth are different from those of term infants. The smaller the gestation the smaller the initial speed of head growth just after birth. The timing of maximum head growth velocity is delayed inversely with gestational age.

The degree of delay is not only defined by the gestational age. Groups $\mathrm{H}$ and $\mathrm{L}$ (gestation 30-33 weeks) were divided according to caloric intake.
Group L (low intake) showed a prolonged delay in achieving maximum OFC velocity while group $\mathbf{H}$ (high intake) showed an early peak with a velocity curve approaching that for near term babies. As this is a retrospective study, no definite conclusions can be drawn for caloric intake, but it may be presumed that neonatal conditions were in general better for group $\mathbf{H}$. This suggests that perhaps under better conditions preterm infants could achieve maximum head growth velocity nearer birth. Analysis of figures from Sher and Brown (1975a) for head growth in infants of 30-33 weeks' gestation show just such an effect, with achievement of maximum velocity soon after birth, the later parts of the curve approximating to our group E.

The above observations suggest that curves of intrauterine head growth are only valuable in assessing OFC at birth. For continued assessment of growth, curves derived from longitudinal data on AFD infants would be more useful. Furthermore, different patterns of head growth velocity may be a result of neonatal insults and the quality of neonatal care. After any significant change in therapy the OFC growth curve for a preterm infant should be reassessed.

Although by the 12th postconceptional month the OFC for preterm infants approaches that of term infants, the way in which this is achieved is different in the two groups (Figs. 9 and 10). The phase of maximum volumetric growth occurs at completely different stages of maturation. There is accumulating evidence, mostly in animals, that disturbance of normal growth at a critical period of brain growth results in permanent impairment of the nervous system and subsequent neurological or behavioural function (Balazs, 1972; Cheek, 1975).

An association between slowed growth of the biparietal diameter with intrauterine growth retardation has been established (Campbell and Newman, 1971). But catch-up growth begins once delivery has taken place. Our curves for postnatal head growth velocity show that the term SFD infants have a similar pattern to the term AFD infants but with a slightly greater initial velocity. This difference persists for the first 5 months of postnatal life but, in spite of this, catch-up growth is not complete by the fifth month.

The postnatal growth patterns of body weight are shown to be largely different to those of OFC growth in that the velocity of weight acquisition accelerates in all groups of babies during the neonatal period with a maximum between 1 and 2 months of age. In term infants it looks as if the head is ready to grow to its maximum soon after birth, whereas weight needs an adaptation period of 5 weeks.

The importance of critical determinants in head 
growth velocity in the neonatal period should be clearly recognized especially with the recent trend in long-term survival of very preterm infants.

We thank Drs. R. Miyake and M. Miyake for allowing us to study the case records of infants under their care, and are grateful to Professor J. P. M. Tizard and Dr. S. J. Oppenheimer for reviewing the manuscript.

\section{References}

Babson, S. G., and Henderson, N. B. (1974). Fetal undergrowth: relation of head growth to later intellectual performance. Pediatrics, 53, 890-894.

Balazs, R. (1972). Effects of hormones and nutrition on brain development. Advances in Experimental Medicine and Biology, 30, 385-415.

Baum, J. D., and Searls, D. (1971). Head shape and size of preterm low-birthweight infants. Developmental Medicine, and Child Neurology, 13, 576-581.

Bray, P. F., Shields, W. D., Wolcott, G. J., and Madsen, J. A. (1969). Occipitofrontal head circumference-an accurate measure of intracranial volume. Journal of Pediatrics, 75, 303-305.

Buda, F. B., Reed, J. C., and Rabe, E. F. (1975). Skull volume in infants. American Journal of Diseases of Children, 129, 1171-1174.

Campbell, S., and Newman, G. B. (1971). Growth of the fetal biparietal diameter during normal pregnancy. Journal of Obstetrics and Gynaecology of the British Commonwealth, 78, 513-519.

Cheek, D. B. (1975). Fetal and Postnatal Cellular Growth. Wiley, London.

Conde, C., Martinez, M., and Ballabriga, A. (1974). Some chemical aspects of human brain development. Pediatric Research, 8, 89-92.

Cruise, M. O. (1973). A longitudinal study of the growth of low birth weight infants. Pediatrics, 51, 620-628.

Davies, P. A., and Davis, J. P. (1970). Very low birthweight and subsequent head growth. Lancet, 2, 1216-1219.

Davies, P. A., and Stewart, A. L. (1975). Low-birth-weight infants: neurological sequelae and later intelligence. British Medical Bulletin, 31, 85-91.

Davies, P. A., and Tizard, J. P. M. (1975) Very low birthweight and subsequent neurological defect. Developmental Medicine and Child Neurology, 17, 3-17.

Dobbing, J. (1974). The later development of the brain and its vulnerability. Scientific Foundations of Paediatrics, p. 565. Ed. by J. A. Davis and J. Dobbing. Heinemann, London.
Dobbing, J., and Sands, J. (1973). The quantitative growth and development of the human brain. Archives of Disease in Childhood, 48, 757-767.

Francis-Williams, J., and Davies, P. A. (1974). Very low birthweight and later intelligence. Developmental Medicine and Child Neurology, 16, 709-728.

Glass, L., Lala, R. V., Jaiswal, V., and Nigam, S. K. (1975). Effect of thermal environment and caloric intake on head growth of low birthweight infants during late neonatal period. Archives of Disease in Childhood, 50, 571-573.

Gruenwald, P. (1967). Growth of the human fetus. Advances in Reproductive Physiology, 2, 279-309.

Hagberg, B., Olow, I., and Hagberg, G. (1973). Decreasing incidence of low birth weight diplegia - an achievement of modern neonatal care? Acta Paediatrica Scandinavica, 62, 199-200.

Lengerke, H. J. (1974). Ein Verfahren zur Korrektur des Kopfumfangsmasses bei atypischer Kopfform. Klinische Paediatrie, 186, 236-242.

Lubchenco, L. O., Hansman, C., and Boyd, E. (1966). Intrauterine growth in length and head circumference as estimated from live births at gestational ages from 26 to 42 weeks. Pediatrics, 37, 403-408.

Muralt, A. von (1972). The developing brain and the damage inflicted by malnutrition. Lipids, Malnutrition and the Developing Brain. Ed. by K. Elliott and J. Knight. Elsevier, Amsterdam.

O'Connell, E. J., Feldt, R. H., and Stickler, G. B. (1965). Head circumference, mental retardation and growth failure. Pediatrics, 36, 62-66.

O'Neill, E. M. (1961). Normal head growth and the prediction of head size in infantile hydrocephalus. Archives of Disease in Childhood, 36, 241-252.

Pryor, H. B., and Thelander, H. (1968). Abnormally small head size and intellect in children. Journal of Pediatrics, 73, 593-598.

Sher, P. K., and Brown, S. B. (1975a). A longitudinal study of head growth in pre-term infants. I. Normal rates of head growth. Developmental Medicine and Child Neurology, 17, 705-710.

Sher, P. K., and Brown, S. B. (1975b). A longitudinal study of head growth in pre-term infants. II. Differentiation between 'catch-up' head growth and early infantile hydrocephalus. Developmental Medicine and Child Neurology, 17, 711-718.

Winick, M., and Rosso, P. (1969). Head circumference and cellular growth of the brain in normal and marasmic children. Journal of Pediatrics, 74, 774-778.

Correspondence to Dr. M. Fujimura, Department of Paediatrics, Yodogawa Christian Hospital, 57 Awaji Honmachi l-chome, Higashi Yodogawa-ku, Osaka, Japan. 